\title{
Organic solar cells based on anthracene-containing PPE- PPVs and non-fullerene acceptors
}

\author{
$\underline{\text { Shahidul Alam }}^{1,2}$, Rico Meitzner ${ }^{1,2}$, Ogechi V. Nwadiaru ${ }^{1,4}$, Christian Friebe ${ }^{1,2}$, Jonathan Cann ${ }^{7}$, \\ Johannes Ahner ${ }^{2}$, Christoph Ulbricht ${ }^{5,6}$, Zhipeng $\mathrm{Kan}^{8}$, Stephanie Höppener ${ }^{2,3}$, Martin D. \\ Hager $^{1,2}$, Daniel A. M. Egbe ${ }^{5,6}$, Gregory C. Welch ${ }^{7}$, Frédéric Laquai ${ }^{8}$, Ulrich S. Schubert ${ }^{1,2}$, and \\ Harald Hoppe ${ }^{1,2}$ \\ ${ }^{1}$ Center for Energy and Environmental Chemistry Jena (CEEC Jena), Friedrich Schiller University Jena, \\ Philosophenweg 7a, D-07743 Jena, Germany \\ ${ }^{2}$ Laboratory of Organic and Macromolecular Chemistry (IOMC), Friedrich Schiller University Jena, Humboldt \\ Straße 10, D-07743 Jena, Germany \\ ${ }^{3}$ Jena Center for Soft Matter (JCSM), Philosophenweg 7, 07743 Jena, Germany \\ ${ }^{4}$ Pan African University, Institute of Water and Energy Sciences (Including Climate Change)-PAUWES, 13000 \\ Tlemcen, Algeria \\ ${ }^{5}$ Linz Institute for Organic Solar Cells (LIOS), Johannes Kepler University Linz, Altenbergerstraße. 69, 4040 Linz, \\ Austria. \\ ${ }^{6}$ Institute of Polymeric Materials and Testing, Johannes Kepler University, Altenbergerstraße. 69, 4040 Linz, \\ Austria \\ ${ }^{7}$ Department of Chemistry, 731 Campus Place N.W., University of Calgary, Canada \\ ${ }^{8}$ Physical Sciences and Engineering Division (PSE), Material Science and Engineering (MSE), Solar and \\ Photovoltaics Engineering Research Center (SPERC), King Abdullah University of Science and Technology \\ (KAUST), Thuwal 23955-6900, Kingdom of Saudi Arabia
}

\begin{abstract}
Lately, non-fullerene acceptors (NFAs) have received increasing attention for use in polymerbased bulk-heterojunction (BHJ) organic solar cells (OSCs), as improved photovoltaic performance compared to classical polymer-fullerene blends could be demonstrated. In this study, polymer solar cells based on a statistically substituted anthracene-containing poly $(p$ phenyleneethynylene)-alt-poly( $p$-phenylenevinylene)s (PPE-PPVs) copolymer (AnE-PVstat) as donor in combination with a number of different electron accepting materials were investigated. Strong photoluminescence quenching of the polymer donor indicates intimate intermixing of both materials. However, the photovoltaic performances were found to be poor compared to blends that use fullerene as acceptor. Time-delayed collection field (TDCF) measurements demonstrate: charge generation is field-independent, but bimolecular recombination processes limit the fill factor and thus the efficiency of devices.
\end{abstract}

\section{Keywords}

Non-fullerene acceptors (NFA), Bulk-heterojunction (BHJ), Organic solar cell (OSC)

\section{Corresponding author}

Shahidul Alam, Dr. Harald Hoppe

E-mail: shahidul.alam@uni-jena.de, harald.hoppe@uni-jena.de 


\section{Introduction}

The increase in the global population is continually putting more and more strain on energy supply, leading to excessive exploitation of conventional energy resources such as fossil fuels. Low cost and abundant energy solutions can release this strain to some extent(Holdren 1991; IEA 2015). Hence, the search for low-cost renewable energy solutions is currently considered top priority in energy research. The deployment of organic photovoltaics (OPV) as a renewable energy technology has shown promise of reducing both, direct technology costs and environmental impact. This technology offers greater ease of fabrication, less energy-intensive production and the possibility roll-to-roll manufacturing (Günes et al. 2007; Krebs et al. 2010; Mulligan et al. 2014; Zhao et al. 2017). In the last decade research on OPVs have seen a steady and fast growth with improved efficiencies now above $13 \%$, obtained with bulk heterojunction OSCs (Zhao et al. 2017). One of its most prominent attractions is the ability to be processed from solution and the applicability of fast mass printing techniques that allow for high production output with relatively low material consumption (Cao and Xue 2014). Fullerene derivatives are one of the most fundamental components in commercially available polymer solar cells. They are used as an acceptor, typically blended with an electron-rich donor to yield the photoactive layer of the solar cells (Hummelen et al. 1995; Wienk et al. 2003). Although, there are quite a number of advantages of applying fullerenes in polymer solar cells, including an energetically low-lying lowest unoccupied molecular orbital (LUMO) (Allemand et al. 1991), ultra-fast charge transfer, and high electron mobility (Singh et al. 2005; Thompson and Fréchet 2008), there commercial application is limited. A high synthesis cost is one of the most critical hurdles preventing the application of fullerene derivatives. Scientifically, impurities created during synthesis, weak absorption in the solar spectral range limiting photon harvesting to the absorption of the donor material (Ren et al. 2011), and chemical and energetic tenability are major issues. Non-fullerene acceptors (NFAs) have recently emerged as replacement of fullerenes, and over the past few years have received the increased attention of a growing research community. Organic based molecular NFAs have developed as excellent materials for use in high-performance bulk heterojunction solar cells (Eftaiha et al. 2014; McAfee et al. 2015; Sonar et al. 2011), some of the best and most studied NFAs are based upon the perylene diimide chromophore (Kozma and Catellani 2013; Liu et al. 2016), and have demonstrated photovoltaic power conversion efficiencies (PCE) as high as 9.5\% (Liang et al. 2017).

The acceptors used in this study are molecular-based materials with an A-D-A structural motif, where $\mathrm{A}$ is an acceptor moiety and $\mathrm{D}$ a donor moiety. This molecular structure allows for a considerable flexibility in molecular design, bandgap engineering, and fine-tuning the absorption spectrum, and blend morphology (Liang et al. 2017; Nielsen et al. 2015). Furthermore, it allows for a broad range of synthetic approaches. Also through the modification of the attached side chains, their solubility in different solvents, as well as the blend morphology, can be tuned. Hence it becomes readily possible to refrain from halogenated solvents (Wadsworth et al. 2017) without losing performance (Dayneko et al. 2017), which was already shown for some polymer:NFA's blends. All these facts promise a bright future for NFA based OSCs (Bicciocchi et al. 2015).

In this work, we present a polymer donor, a PPE-PPV derivative called AnE-PVstat, in combination with NFAs that show a complementary absorption spectrum. All blends showed strong quenching of photoluminescence but unfortunately no or only a deficient photovoltaic performance. The reasons behind the poor photovoltaic performance will be elucidated in the following. 


\section{Experimental}

\section{Materials}

Polymer:NFA blend solutions were prepared from 2:3 (mass\% ratio) mixtures. The synthesis of AnE-PVstat is described elsewhere (Egbe et al. 2010; Kivrak et al. 2017a; Kivrak et al. 2017b). In order to compare with a standard acceptor material, PCBM (Solenne) was used as received. The chemical structure of AnE-PVstat, PCBM and NFAs are shown in

Figure 1. The synthesis of ITIC and PDI based non-fullerene acceptors were described elsewhere (Cann et al. 2017a; Cann et al. 2017b; Lin et al. 2015; McAfee et al. 2017).

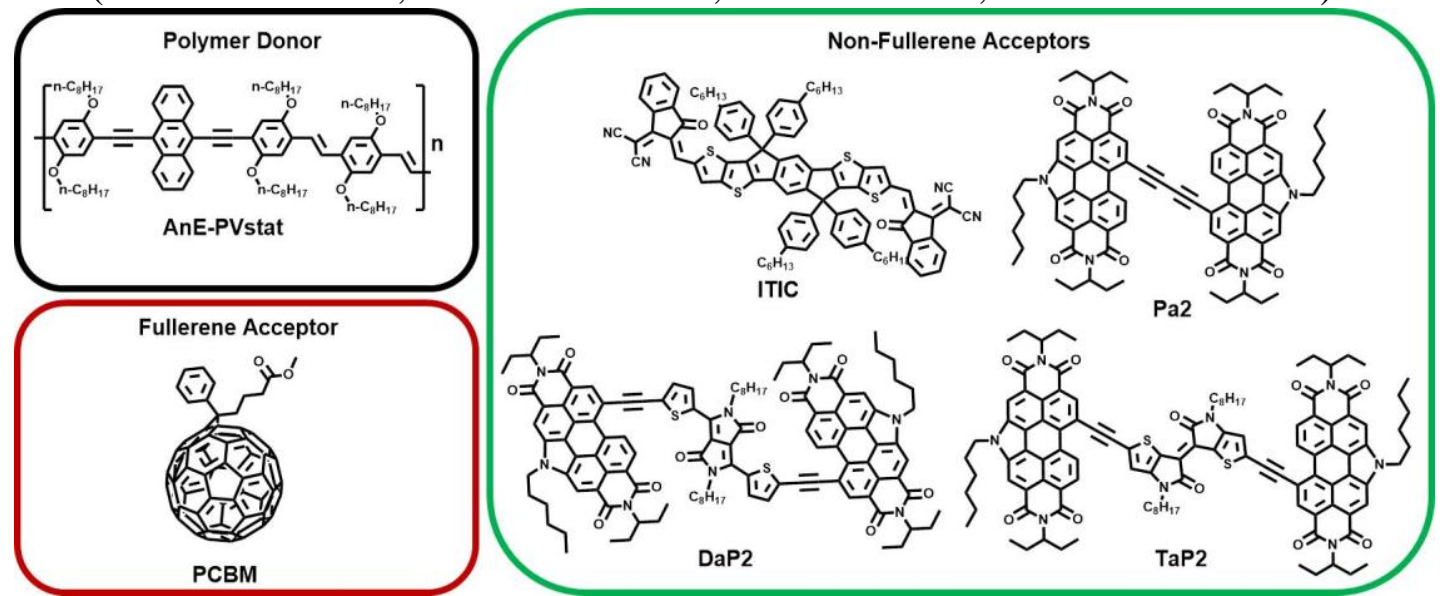

Figure 1: Molecular structure of AnE-PVstat $\left(\mathrm{C}_{8} \mathrm{H}_{17}=n\right.$-octyl or 2-ethylhexyl), PCBM, ITIC $\left(\mathrm{C}_{6} \mathrm{H}_{13}=n\right.$-hexyl), $\mathrm{PDI}_{2} \mathrm{Ac}_{2}(\mathbf{P a} 2)\left(\mathrm{C}_{6} \mathrm{H}_{13}=n\right.$-hexyl $), \mathrm{DPP}-(\mathrm{Ac}-\mathrm{PDI})_{2}(\mathbf{D a P 2})\left(\mathrm{C}_{6} \mathrm{H}_{13}=n\right.$-hexyl, $\mathrm{C}_{8} \mathrm{H}_{17}=2$-ethylhexyl $)$ and TII-(Ac$\mathrm{PDI})_{2}(\mathbf{T a P 2})\left(\mathrm{C}_{6} \mathrm{H}_{13}=n\right.$-hexyl, $\mathrm{C}_{8} \mathrm{H}_{17}=2$-ethylhexyl $)$.

The donor material is a side chain based statistical copolymer with a random distribution of segments of linear octyloxy side chains and of branched 2-ethylhexyloxy side chains on an anthracene-containing poly( $p$-phenylene-ethynylene)-alt-poly( $p$-phenylene-vinylene) (PPE-PPV) backbone known as AnE-PVstat polymer. The synthesis of this polymer was described in detail in (Egbe et al. 2010), the fullerene acceptor used is a derivative of $\mathrm{C}_{60}$ known as PCBM. The NFA, ITIC is based on the bulky seven-ring fused core (indacenodithieno [3,2-b]thiophene, IT), end-capped with 2-(3-oxo-2,3-dihydroinden-1-ylidene) malononitrile (INCN) groups, and with four 4-hexylphenyl groups substituted on it. From the original publication on the synthesis of ITIC, it is reported to have strong and broad absorption, low-lying LUMO and highest occupied molecular orbital (HOMO) energy levels, good electron transport ability, and good miscibility with polymer donors, the synthesis of this material is detailed previously (Lin et al. 2015). Three additional NFAs derived from perylene diimide (PDI) dimers were investigated. The first $\mathrm{PDI}_{2} \mathrm{Ac}_{2}$ (Pa2) was synthesized by connecting two PDI monomers with a double acetylene bridge. The second DPP-(Ac-PDI) 2 (DaP2) is obtained by a synthesis of diketopyrrolopyrrole (DPP) connected to the two molecules of PDI by an acetylene bridge on each side. DPP based materials have been so far investigated for their excellent charge carrier mobilities and fluorescent properties. The third acceptor was based on thienoisoindigo (TII) and acetylene (TaP2), which serves as the linker moiety between the PDI dimers, based on that earlier representative derived from thienoisoindigo have shown good optical properties and charge carrier mobilities but have not been appropriately investigated in OSC applications. The schematics of all materials used for this study are given in Figure 1.

\section{Electrochemical characterization}

To match the energy levels in organic photovoltaic devices, it is essential to determine the HOMO and LUMO onsets of the donor and acceptor components. Cyclic voltammetry (CV) is a powerful tool to determine the HOMO and LUMO energy of organic elements. The ionization 
potential (HOMO) level and electron affinity (LUMO) level can be estimated from oxidation and reduction onset potentials on the basis of a redox reference such as ferrocene $(5.0 \mathrm{eV}$ below the vacuum level) (Namazian et al. 2010).

$$
\begin{gathered}
\mathrm{E}_{\mathrm{HOMO}}=-\left(\left(\mathrm{E}_{\mathrm{ox}}\right)_{\text {onset }}+\text { potential of } \mathrm{Fc} / \mathrm{Fc}^{+} \text {couple }\right) \mathrm{eV} \\
\mathrm{E}_{\mathrm{LUMO}}=-\left(\left(\mathrm{E}_{\mathrm{red}}\right)_{\text {onset }}+\text { potential of } \mathrm{Fc} / \mathrm{Fc}^{+} \text {couple }\right) \mathrm{eV}
\end{gathered}
$$

The HOMO and LUMO values were evaluated from the first oxidation and reduction peak onsets of the $\mathrm{CV}$, the measurements were performed on a VMP3 potentiostat (Biologic, France) using a standard three-electrode setup with a glassy-carbon disk working electrode (diameter $2 \mathrm{~mm}$ ), a $\mathrm{AgNO}_{3} / \mathrm{Ag} / \mathrm{CH}_{3} \mathrm{CN}$ reference electrode and a platinum wire counter electrode. The measurements were conducted in chloroform (spectroscopic grade) containing $0.1 \mathrm{M} \mathrm{Bu}_{4} \mathrm{NPF}_{6}$ as conducting salt and ca. $1 \mathrm{mg} \cdot \mathrm{mL}^{-1}$ of the sample. The solutions were de-aerated via purging with argon prior to each measurement. The applied scan rate was $200 \mathrm{mV} \cdot \mathrm{s}^{-1}$.

\section{Optical characterization}

Contactless and non-destructive thin film steady-state photoluminescence (PL) spectra were recorded with an AvantesAvaSpec ULS-2048 fiber spectrometer. PL excitation was conducted with a laser diode emitting at $405 \mathrm{~nm}$. For PL correction of absorption and evaluation of optical band-gaps, thin film transmission and reflection spectra were recorded with two Avantes AvaSpec-ULS3648-USB2-UA-25 fiber spectrometers reassembled to the thin film absorption spectra.

\section{Atomic Force Microscopy (AFM)}

To gain further insight into the morphology of the blends system, AFM measurements were performed in tapping mode on a NTEGRA Aura (NT-MDT) using tapping mode cantilevers from Mikromasch (NSC35).

\section{I-V characterization}

Photovoltaic devices of conventional and inverted architecture were studied under dark and AM1.5 conditions. The current-voltage (IV) measurements of the solar cell devices were recorded with a Keithley 2400 Source-Meter-Unit (SMU). The intensity of the light source was calibrated by a standard silica photodiode to confirm AM1.5 condition and the intensity of 100 $\mathrm{mW} / \mathrm{cm}^{2}$. The details of the solar cells preparation and layer stacks were explicated in the experimental section.

\section{Time-Delayed Collection Field (TDCF)}

TDCF is a pump-probe technique, and the analyses that are particularly relevant to the study of field dependence of charge generation, charge extraction, and charge recombination dynamics in solar cells (Albrecht et al. 2012; Kniepert et al. 2014; Kniepert et al. 2011).The home-built TDCF setup uses the second harmonic $(532 \mathrm{~nm})$ of an actively Q-switched sub-ns Nd:YVO4 laser (InnoLas picolo AOT) operating at $5 \mathrm{kHz}$ as excitation. In order to minimize the RC response time, a small device area of $1 \mathrm{~mm}^{2}$ was used. The samples were measured under dynamic vacuum conditions to avoid any degradation. A Keysight S1160A functional generator was used to provide the pre-bias and extraction bias, while a Keysight four-channel digital oscilloscope was used to measure the current response of the device.

\section{Preparation of films and BHJ OSCs}

The global polymer and acceptors weight ratio was held constant at 2:3. AnE-PVstat and the acceptor materials were dissolved in a 1:1 (vol \%) solvent mixture of chlorobenzene and chloroform $(\mathrm{CB}: \mathrm{CF})$ as found to be the optimal mixture promoting phase separation via aggregation in AnE-PVstat:PCBM blends (Kastner et al. 2015; Kastner et al. 2013). However, the solvent systems were not optimized for non-fullerene acceptors. $12 \mathrm{mg} / \mathrm{mL}$ polymer and 18 $\mathrm{mg} / \mathrm{mL}$ of acceptor were prepared separately. In order to have 2:3 blend ratio of polymer (12 $\mathrm{mg} / \mathrm{mL})$ :acceptor $(18 \mathrm{mg} / \mathrm{mL})$ was blended together in 1:1 ratio from the pristine solutions. The 
films were casted from pristine and blended solution onto glass for optical measurements. Blended solutions were coated as active layer of OSC.

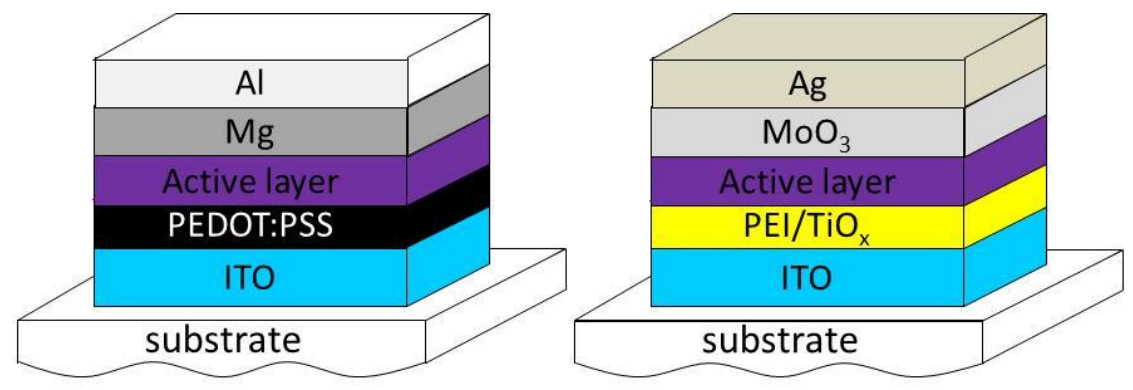

Figure 2: Schematic representation of the conventional (left) and inverted (right) polymer solar cell layer stacks.

For fabrication of the OSC, two different layer stack architectures were used. The fabrication of OSC started with cleaning the glass/indium tin oxide (ITO) substrates in ultrasonic bath successively using acetone and isopropanol for $15 \mathrm{~min}$ each. In the case of conventional architecture, poly(3,4ethylenedioxythiophene)-poly(styrene sulfonate) (PEDOT:PSS) was spincasted from aqueous solution on top of ITO at $3000 \mathrm{rpm}$ for 35 seconds as the hole transporting layer (HTL). The substrates were then annealed on a hot plate for 15 minutes at $180^{\circ} \mathrm{C}$ in order to remove the residual water content from the films. Afterward, the samples were transferred to the glovebox $\left(\mathrm{N}_{2}\right)$ immediately and cooled down to room temperature. Active layer solutions were casted on top of the PEDOT:PSS layer with $600 \mathrm{rpm}$ spin frequency and run for 45 seconds. Later on the films were transferred to the vacuum chamber in order to deposit the top electrode. Solar cells were finished with thermal evaporation of $50 \mathrm{~nm}$ of magnesium and 100 $\mathrm{nm}$ of aluminum (Al) deposited sequentially through a shadow mask as an electron collecting electrode giving $0.42 \mathrm{~cm}^{2}$ active area of the solar cells. To build inverted solar cells, polyethyleneimine (PEI)/TiOx blends were spin casted on cleaned ITO substrate and these devices were completed with $10 \mathrm{~nm}$ molybdenum trioxide $\left(\mathrm{MoO}_{3}\right)$ and $100 \mathrm{~nm}$ silver $(\mathrm{Ag})$ through a shadow mask. $0.27 \mathrm{mg} / \mathrm{ml}$ PEI is diluted in Butanol and $1.5 \mathrm{vol} \%$ TiOx in Isopropanol are blended with the ratio of 1:1 (vol \%). PEI and TiOx blends are spin-coated on top of the ITO at $3000 \mathrm{rpm}$ for 30 seconds. Thin layer of PEI/TiOx was annealed at $105^{\circ} \mathrm{C}$ for 5 min inside the glove-box. Photoactive layers were kept the same as in conventional architecture. All the OSCs were sealed under glass using UV-curing glue (epoxy-based) inside the glove box. Conventional and inverted architectures are shown in Figure 2.

\section{Results and Discussion}

In order to elucidate the energy alignment and compatibility of the AnE-PVstat polymer with different non-fullerene acceptors, CV measurements were performed on pristine materials. In case of AnE-PVstat, we can see a first oxidation wave at $\mathrm{E}_{\text {onset }}=0.25 \mathrm{~V}$, a second one, which leads to subsequent irreversible reactions (most likely electro-polymerization), and a reduction signal at $\mathrm{E}_{\text {onset }}=-1.24 \mathrm{~V}$ were obtained. We note that the latter two signals are electrochemically irreversible. However, all the non-fullerene acceptors show quasi-reversible oxidation signals. And ITIC showed an electrochemically irreversible reduction signal at $\mathrm{E}_{\text {onset }}=-1.04 \mathrm{~V}$. In contrast; all the PDI-based non-fullerene acceptors exhibited quasi-reversible reduction signals. Figure 3 illustrates the CV curves of AnE-PVstat, PCBM, ITIC, Pa2, DaP2, TaP2, in detail oxidation and reduction onset values are presented in the graph. 


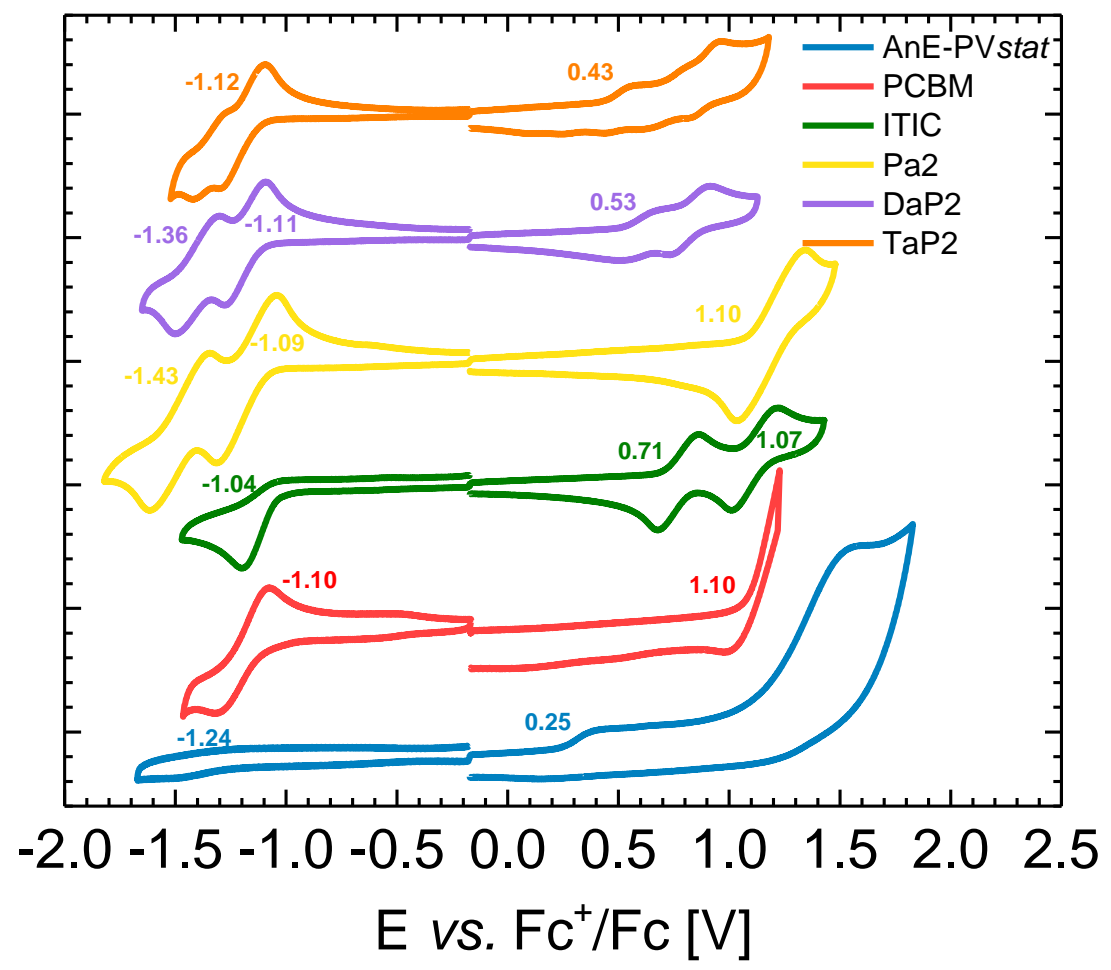

Figure 3: CV curves of AnE-PVstat, PCBM, ITIC, Pa2, DaP2 and TaP2, oxidation and reduction onsets are presented in the graph.

Bandgap energies were calculated from the HOMO-LUMO difference. Table 1 summarizes the electrochemical data obtained from the CV measurements. The expected energy level alignment in type II heterojunctions can be estimated from Figure 4.

Table 1: Oxidation and reduction onset potentials from CV measurements, calculated HOMO, LUMO energies for the pristine polymer and different acceptor materials as well as their electrochemical and optical (see below) bandgaps and their difference are summarized.

\begin{tabular}{|c|c|c|c|c|c|c|c|c|c|}
\hline Material & \multicolumn{2}{|c|}{ Oxidation onset } & \multicolumn{2}{|c|}{ Reduction onset } & \multirow[t]{2}{*}{$\begin{array}{c}\mathrm{HOMO} \\
(\mathrm{eV})\end{array}$} & \multirow[t]{2}{*}{$\begin{array}{c}\text { LUMO } \\
(\mathrm{eV})\end{array}$} & \multirow[t]{2}{*}{$\begin{array}{c}\text { Electrochemical } \\
\text { bandgap } \mathrm{E}_{\mathrm{G}, \mathrm{EC}}(\mathrm{eV})\end{array}$} & \multirow{2}{*}{$\begin{array}{l}\text { Optical bandgap } \\
\mathrm{E}_{\mathrm{G}, \mathrm{O}} *\end{array}$} & \multirow{2}{*}{$\begin{array}{c}\Delta \mathrm{E}_{\mathrm{G}}=\quad \mathrm{E}_{\mathrm{G}, \mathrm{O}} \\
-\mathrm{E}_{\mathrm{G}, \mathrm{EC}}\end{array}$} \\
\hline AnE_Pystat & $\frac{1}{025}$ & 2 & $\begin{array}{r}1 \mathrm{St} \\
-124\end{array}$ & & & & & & \\
\hline & & & & & & & & & \\
\hline РСВМ & 1.10 & & -1.10 & & -6.10 & -3.90 & 2.20 & 2.33 & 0.13 \\
\hline ITIC & 0.71 & 1.07 & -1.04 & & -5.71 & -3.96 & 1.75 & 1.45 & -0.30 \\
\hline $\mathrm{Pa} 2$ & 1.10 & & -1.09 & -1.43 & -6.10 & -3.91 & 2.19 & 1.94 & -0.25 \\
\hline DaP2 & 0.53 & & -1.11 & -1.36 & -5.53 & -3.89 & 1.64 & 1.50 & -0.14 \\
\hline TaP2 & 0.43 & & -1.12 & & -5.43 & -3.88 & 1.55 & 1.33 & -0.22 \\
\hline
\end{tabular}

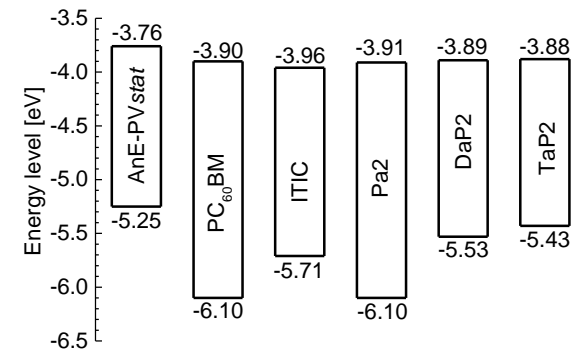

Figure 4: HOMO-LUMO energy levels, respectively bandgaps, of AnE-PVstat, PCBM, ITIC, Pa2, DaP2 and TaP2 are depicted. 
Thin films of AnE-PVstat:PCBM and AnE-PVstat:NFA blends on glass substrates were processed from solution at room temperature under inert $\left(\mathrm{N}_{2}\right)$ environment. These samples were used in spectroscopic and morphology studies. The obtained absorption and photoluminescence spectra are shown in Figure 5.
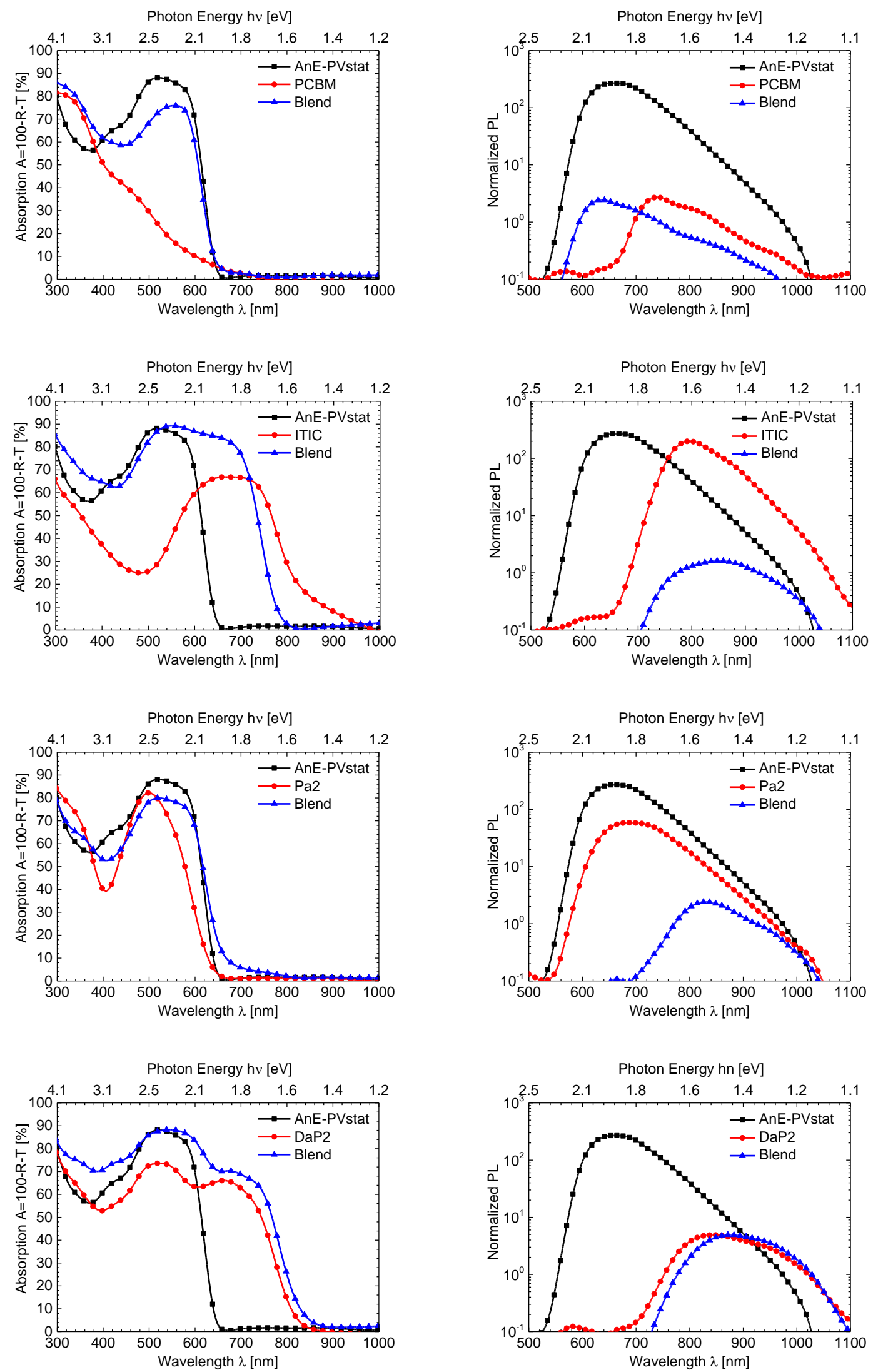

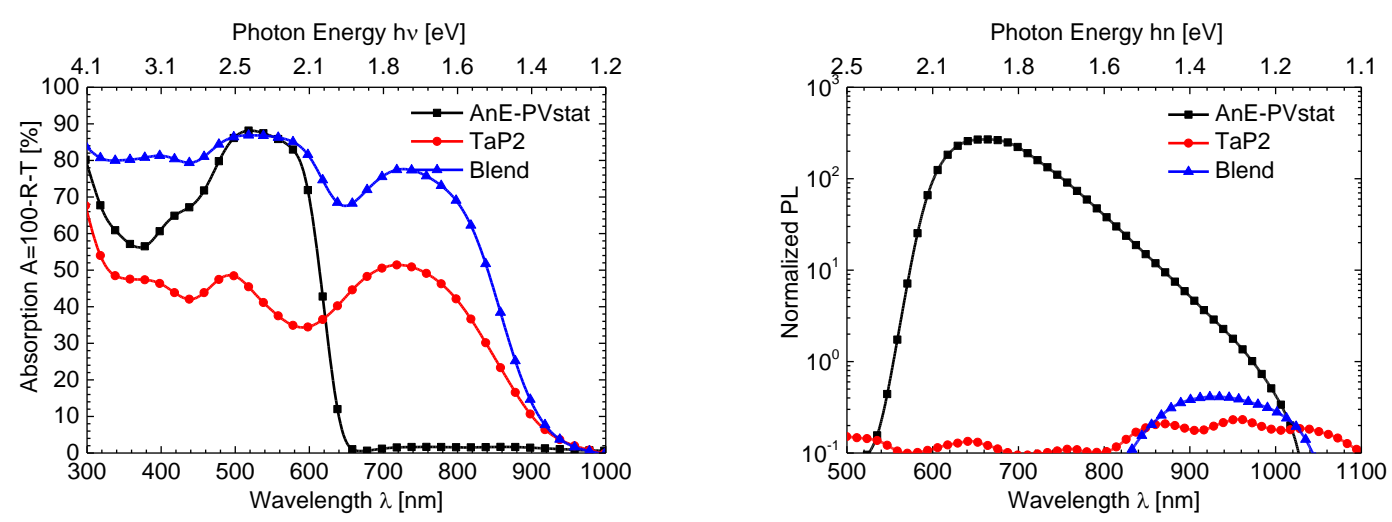

Figure 5: Absorption (left hand side) and photoluminescence (right hand side) spectra for AnE-PVstat, PCBM and various NFA's, in pristine films and blends.

Pristine AnE-PVstat donor, pristine acceptors and blends thereof are compared in absorption and PL. On the left-hand side of Figure 5, absorption spectra of the pristine AnE-PVstat, pristine acceptors, and their blends are shown. DaP2 and TaP2 show a complimentary absorption to AnE-PVstat increasing the absorption range, while Pa2 only slightly adds some absorption towards longer wavelengths to the blend with AnE-PVstat. Optical band gaps are extracted from the intersection between the absorption and PL spectrum of pristine materials for comparison with electrochemical band gaps. The values of the optical band gap $\left(E_{\mathrm{g}}\right)$ and the difference with respect to electrochemical gaps are shown in Table 1.

In order to compare the PL intensities from the thin film samples, the recorded PL spectra have been normalized with respect to the absorption at the excitation wavelength of the laser (405 $\mathrm{nm})$. On the right hand side of Figure 5, the PL spectra of pristine AnE-PVstat, pristine acceptors, and their blends are shown. From the PL spectra of the AnE-PVstat:PCBM, we can deduce that the polymer PL is noticeably quenched, the quenching factor is $\sim 110$. According to the PL results, AnE-PVstat:NFA's blends may be so finely intermixed that grain sizes become too small and thus PL signals are quenched.

The PL intensity of pristine AnE-PVstat and ITIC in films are more or less same; however polymer intensity is completely quenched in the blend. A PL spectrum of Pa2 is similar to AnEPVstat. However the polymer PL is quenched and we could only see the acceptor PL in the blend. In case of DaP2 and TaP2, strong quenching of all PL signals upon blending with AnEPVstat is found. We can conclude from the PL studies that there are sufficient energy level offsets for electron transfer. The energy transfer could occur for ITIC, DaP2, and TaP2 as the optical absorption overlaps with the emission of the polymer donor.
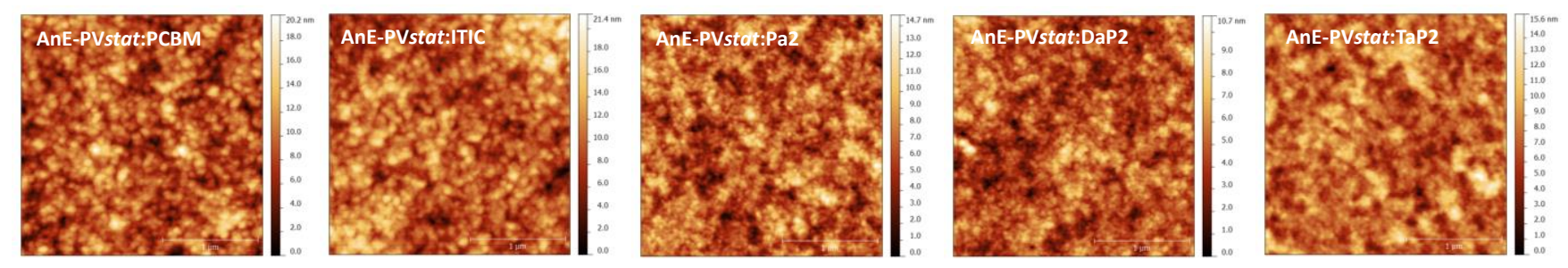

Figure 6: Tapping mode AFM images (area 2.5 x $2.5 \mu \mathrm{m} 2$ ) are shown for AnE-PVstat:PCBM and AnEPVstat:NFA's blends.

AFM measurements were conducted on the same films that were used for optical characterization are shown in Figure 6. The AFM images show that in case of PCBM and ITIC, 
there is a slightly larger average grain size, which may be associated with the AnE-PVstat phase. Such a larger phase separation may yield more favorable charge percolation pathways than for the remaining non-fullerene acceptors. The smaller apparent domain sizes are in good agreement with complete polymer PL quenching. Although AFM topography images do not afford adequate in-depth information about the blend morphology for PDI based NFA's with AnE-PVstat, the most straightforward assumptions are no changes within the blend organization occur in the depth of the film. This leads finely scaled surface morphology due to the homogeneously intermixed bulk structure.

Figure 7 illustrates dark (left) and light (right) I-V curves of the solar cells prepared in conventional and inverted architectures. In case of conventional architecture, only blends with PCBM exhibit a decent diode characteristic in the dark. The remaining blends show symmetric characteristics. Although, when recorded under solar simulation, a somewhat increased current was found for all blends, the currents remained more or less symmetric - but now around the open circuit voltage for blends with NFA's. Alone AnE-PVstat:PCBM blends exhibited the required photovoltaic characteristics.

In case of inverted devices, the situation became even more remarkable, while for PCBM the diode characteristics improved even further, leading to a higher rectification than for the conventional architecture, specifically the DaP2 and TaP2 exhibited shallow currents within the ohmic regime. However, under forward bias diode characteristics could be observed in all cases, though differently well developed. In general, lower photocurrents were obtained for inverted devices but somewhat increased open circuit voltages were found for all NFA-based blends. Table 2 shows the summary of the photovoltaic parameters of the solar cells devices of conventional and inverted architecture.
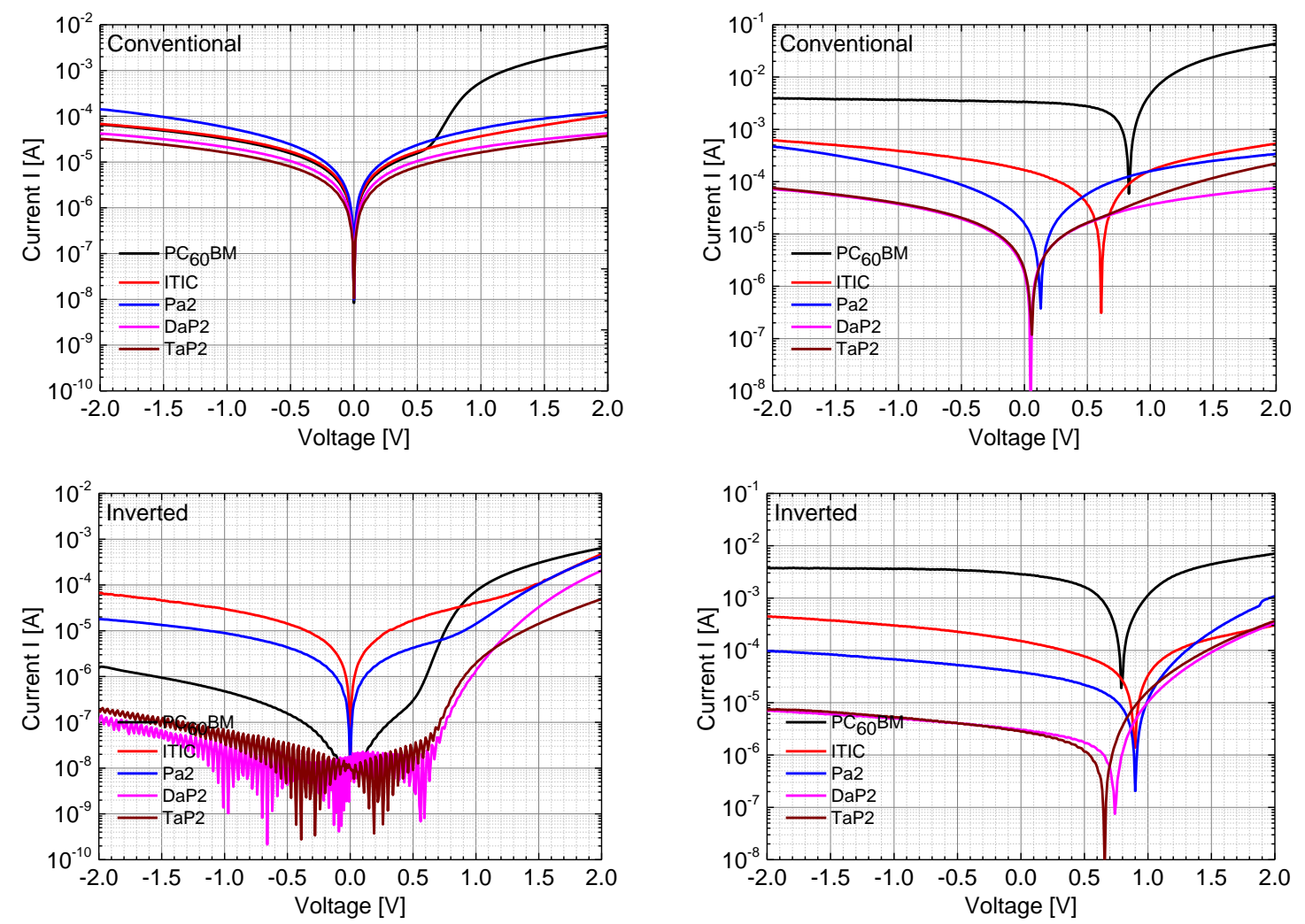

Figure 7: Top-left: logarithmic dark I-V curves of conventional solar cells, top-right: logarithmic I-V curves of conventional solar cells under illumination. Bottom-left: logarithmic dark I-V curves of inverted solar cells, bottomright: logarithmic I-V curves of inverted solar cells under illumination. 
Table 2: Photovoltaic parameters from solar cells devices of conventional and inverted configuration

\begin{tabular}{|c|c|c|c|c|c|c|c|}
\hline Materials & Solar cell type & $\mathrm{J}_{\mathrm{SC}}\left(\mathrm{mA} / \mathrm{cm}^{2}\right)$ & $\mathrm{V}_{\mathrm{OC}}(\mathrm{mV})$ & $\mathrm{FF}(\%)$ & $\eta(\%)$ & $\mathrm{R}_{\mathrm{S}}(\mathrm{Ohm})$ & $\mathrm{R}_{\mathrm{P}}(\mathrm{kOhm})$ \\
\hline AnE-PVstat:PCBM & Conv. & 7.951 & 830 & 53 & 3.48 & 26 & 2.4 \\
\hline AnE-PVstat:ITIC & Conv. & 0.396 & 612 & 28 & 0.07 & 2332 & 4.4 \\
\hline AnE-PVstat:Pa2 & Conv. & 0.038 & 127 & 25 & $<0.01$ & 5297 & 8.1 \\
\hline AnE-PVstat $:$ DaP2 & Conv. & 0.004 & 50 & 24 & $<0.01$ & 25095 & 28.4 \\
\hline AnE-PVstat:TaP2 & Conv. & 0.005 & 57 & 25 & $<0.01$ & 4359 & 27.2 \\
\hline AnE-PVstat:PCBM & Inv. & 6.824 & 795 & 36 & 1.97 & 181 & 0.64 \\
\hline AnE-PVstat $:$ ITIC & Inv. & 0.359 & 895 & 29 & 0.09 & 3267 & 6.7 \\
\hline AnE-PVstat:Pa2 & Inv. & 0.091 & 900 & 32 & 0.03 & 347 & 34.3 \\
\hline AnE-PVstat $:$ DaP2 & Inv. & 0.007 & 739 & 39 & $<0.01$ & 1645 & 471.6 \\
\hline AnE-PVstat:TaP2 & Inv. & 0.007 & 655 & 36 & $<0.01$ & 1544 & 388.1 \\
\hline
\end{tabular}

In order to clarify the origin of low photocurrents, time-delayed collection field (TDCF) measurements were performed on AnE-PVstat:PCBM, AnE-PVstat:ITIC and AnE-PVstat:Pa2 based solar cells in conventional device architecture. Systems that suffer from field-dependent generation, show a strong dependence of the amount of charges generated on the applied electric field, which decreases towards the open circuit voltage, typically leading to low fill factors (Howard et al. 2014) and suffering both geminate and non-geminate recombination, while systems with field-independent charge generation only suffer from non-geminate recombination.
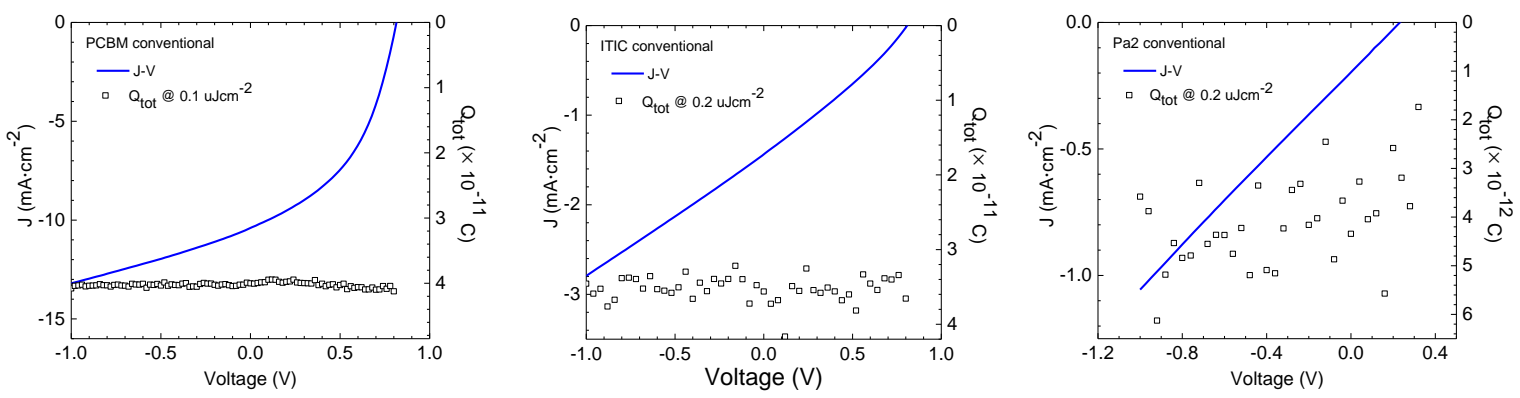

Figure 8: The TDCF data reveals field independent charge generation in AnE-PVstat:PCBM, AnE-PVstat:ITIC and AnE-PVstat:Pa2 solar cells. The sample measured were prepared in conventional architecture

In the present case, low excitation fluence was chosen, to avoid non-geminate recombination losses prior to charge extraction. The total number of charges, namely $\mathrm{Q}_{\text {tot, }}$ generated in the solar cells under these low fluence conditions, is thus a direct measure of the amount of free charge generated by the excitation laser pulse as a function of the applied pre-bias. The measured $\mathrm{Q}_{\text {tot }}$ as a function of the applied pre-bias during photoexcitation, after a delay time of $10 \mathrm{~ns}$ is shown in Figure 8. 

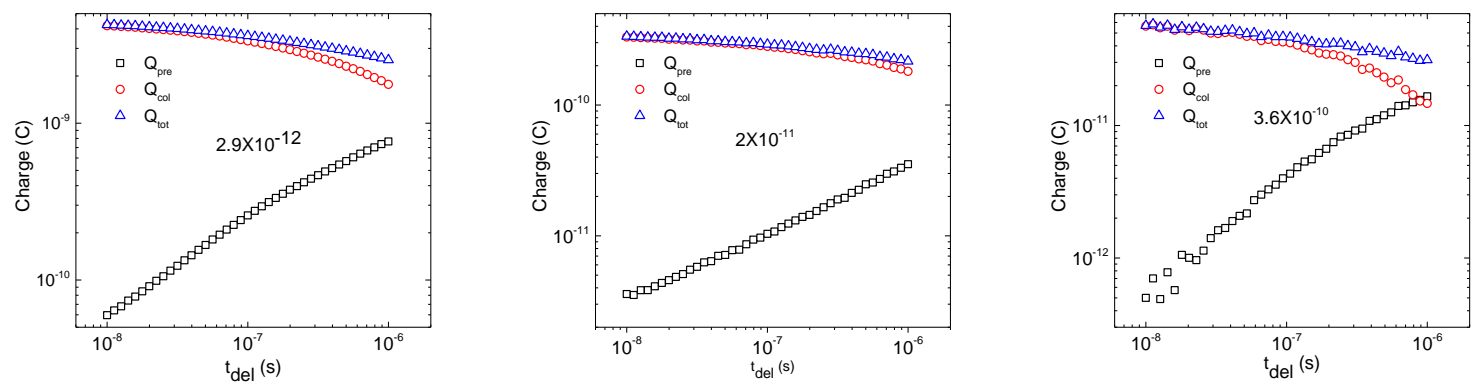

Figure 9: Delay-time charge collection measurements data for AnE-PVstat:PCBM, AnE-PVstat:ITIC and AnE-

PVstat:Pa2 solar cells

In Figure 8, the open squares denote $\mathrm{Q}_{\text {tot }}$ from TDCF measurements. The area above the symbols and below the $\mathrm{J}-\mathrm{V}$ curve (blue line) indicates losses caused by non-geminate (bimolecular) recombination. Charge generation is field-independent in the case of AnEPVstat:PCBM and AnE-PVstat:ITIC devices, suggesting that significant loss in these systems are non-geminate recombination. Because of the low current density in the AnE-PVstat:Pa2 device, the $\mathrm{Q}_{\text {tot }}$ is widely scattered, and does not show a clear trend. It is worth noting that the geminate recombination of CT-states, which do not separate under the low pre-bias applied in TDCF experiments cannot be detected here. To get access to the charge recombination rate, time delayed charge collection measurements were performed is shown in Figure 9. All samples were kept at $\mathrm{V}_{\mathrm{oc}}$, and a low fluence of $4 \mu \mathrm{J} / \mathrm{cm}^{2}$ was chosen. The recombination rate, namely $\mathrm{r}$, was extracted based on fitting to the following equation (Kniepert et al. 2011).

$$
q_{\text {coll }}\left(t_{d}+\Delta t\right)-q_{\text {coll }}\left(t_{d}\right)=-\left[q_{\text {pre }}\left(t_{d}+\Delta t\right)-q_{\text {pre }}\left(t_{d}\right)\right]-\gamma \frac{q_{\text {coll }}^{2}\left(t_{d}\right)}{e A d} \Delta t
$$

The recombination rates for the three measured systems were $2.9 \times 10^{-12}, 2 \times 10^{-11}$, and $3.6 \times 10^{-10}$ $\mathrm{cm}^{3} / \mathrm{s}$, for AnE-PVstat:PCBM, AnE-PVstat:ITIC and AnE-PVstat:Pa2 based solar cells respectively. The recombination rate is massively enhanced in case of NFA's by one and two orders of magnitude, for ITIC and Pa2, respectively.

\section{Conclusion}

In summary, while the moderate performance of organic bulk heterojunction solar cells was obtained for the PPE-PPV copolymer donor combined with the classical fullerene acceptor PCBM, poor performance was found for blends with novel non-fullerene acceptors. Photoluminescence quenching measurements and atomic force microscopy indicated a too fine intermixing of donor and acceptor components. While this alone does not explain the poor photocurrents, strongly increased charge recombination rates were found in case of non-fullerene acceptors via TDCF measurements. Overall, the poor performance of AnE-PVstat combined with NFA's has to be assigned to both, insufficient charge generation and increased charge recombination via non-geminate recombination. The origin of low charge generation yields is currently subject of further studies. This study demonstrates the relative ease of obtaining moderately well-performing fullerene-acceptor based polymer solar cells, while NFAs may require further optimization before they can be successfully matched with existing donor polymers. 


\section{Acknowledgment}

SA, VON and HH are grateful for financial support via DFG in the frame of "PhotoGenOrder".

\section{References}

Albrecht S et al. (2012) On the Field Dependence of Free Charge Carrier Generation and Recombination in Blends of PCPDTBT/PC70BM: Influence of Solvent Additives Journal of Physical Chemistry Letters 3:640-645 doi:10.1021/jz3000849

Allemand P et al. (1991) Two different fullerenes have the same cyclic voltammetry J Am Chem Soc 113:1050-1051

Bicciocchi E, Haeussler M, Rizzardo E, Scully AD, Ghiggino KP (2015) Donor-Acceptor Rod-Coil Block Copolymers Comprising Poly[2,7-(9,9-dihexylfluorene)-alt-bithiophene] and Fullerene as Compatibilizers for Organic Photovoltaic Devices Journal of Polymer Science Part a-Polymer Chemistry 53:888-903 doi:10.1002/pola.27514

Cann J, Dayneko S, Sun JP, Hendsbee AD, Hill IG, Welch GC (2017a) N-Annulated perylene diimide dimers: acetylene linkers as a strategy for controlling structural conformation and the impact on physical, electronic, optical and photovoltaic properties Journal of Materials Chemistry C 5:2074-2083 doi:10.1039/c6tc05107c

Cann JR, Cabanetos C, Welch GC (2017b) Spectroscopic Engineering toward Near-Infrared Absorption of Materials Containing Perylene Diimide ChemPlusChem 82:1359-1364 doi:10.1002/cplu.201700502

Cao WR, Xue JG (2014) Recent progress in organic photovoltaics: device architecture and optical design Energy Environ Sci 7:2123-2144 doi:10.1039/c4ee00260a

Dayneko SV, Hendsbee AD, Welch GC (2017) Fullerene-free polymer solar cells processed from nonhalogenated solvents in air with PCE of 4.8\% Chemical Communications 53:1164-1167 doi:10.1039/c6cc08939a

Eftaiha AF, Sun JP, Hill IG, Welch GC (2014) Recent advances of non-fullerene, small molecular acceptors for solution processed bulk heterojunction solar cells Journal of Materials Chemistry A 2:12011213 doi:10.1039/c3ta14236a

Egbe DAM et al. (2010) Improvement in carrier mobility and photovoltaic performance through random distribution of segments of linear and branched side chains Journal of Materials Chemistry 20:9726-9734 doi:10.1039/COJM01482F

Günes S, Neugebauer H, Sariciftci NS (2007) Conjugated polymer-based organic solar cells Chem Rev 107:1324-1338

Holdren JP (1991) Population and the energy problem Population and Environment 12:231-255 doi:10.1007/bf01357916

Howard IA, Etzold F, Laquai F, Kemerink M (2014) Nonequilibrium Charge Dynamics in Organic Solar Cells Advanced Energy Materials 4:9 doi:1301743

10.1002/aenm.201301743

Hummelen JC, Knight BW, LePeq F, Wudl F, Yao J, Wilkins CL (1995) Preparation and characterization of fulleroid and methanofullerene derivatives Journal of Organic Chemistry 60:532-538

IEA (2015) World Energy Outlook, International Energy Agency 9 rue de la Fédération 75739 Paris Cedex 15, France

Kastner C, Egbe DAM, Hoppe H (2015) Polymer aggregation control in polymer-fullerene bulk heterojunctions adapted from solution Journal of Materials Chemistry A 3:395-403 doi:10.1039/c4ta04736b

Kastner C, Muhsin B, Wild A, Egbe DAM, Rathgeber S, Hoppe H (2013) Improved phase separation in polymer solar cells by solvent blending Journal of Polymer Science Part B-Polymer Physics 51:868-874 doi:10.1002/polb.23286 
Kivrak A, Calis H, Topal Y, Kivrak H, Kus M (2017a) Synthesis of thiophenyl-substituted unsymmetrical anthracene derivatives and investigation of their electrochemical and electrooptical properties Solar Energy Materials and Solar Cells 161:31-37 doi:10.1016/j.solmat.2016.11.006

Kivrak A, Er OF, Kivrak H, Topal Y, Kus M, Camlisoy Y (2017b) Synthesis and solar-cell applications of novel furanyl-substituted anthracene derivatives Opt Mater 73:206-212 doi:10.1016/j.optmat.2017.08.014

Kniepert J, Lange I, van der Kaap NJ, Koster LJA, Neher D (2014) A Conclusive View on Charge Generation, Recombination, and Extraction in As-Prepared and Annealed P3HT:PCBM Blends: Combined Experimental and Simulation Work Advanced Energy Materials 4 doi:1301401

10.1002/aenm.201301401

Kniepert J, Schubert M, Blakesley JC, Neher D (2011) Photogeneration and Recombination in P3HT/PCBM Solar Cells Probed by Time-Delayed Collection Field Experiments Journal of Physical Chemistry Letters 2:700-705 doi:10.1021/jz200155b

Kozma E, Catellani M (2013) Perylene diimides based materials for organic solar cells Dyes and Pigments 98:160-179 doi:10.1016/j.dyepig.2013.01.020

Krebs FC, Nielsen TD, Fyenbo J, Wadstrøm M, Pedersen MS (2010) Manufacture, integration and demonstration of polymer solar cells in a lamp for the "Lighting Africa" initiative Energy Environ Sci 3:512-525

Liang N, Jiang W, Hou J, Wang Z (2017) New developments in non-fullerene small molecule acceptors for polymer solar cells Materials Chemistry Frontiers 1:1291-1303 doi:10.1039/C6QM00247A

Lin Y, Wang J, Zhang Z-G, Bai H, Li Y, Zhu D, Zhan X (2015) An Electron Acceptor Challenging Fullerenes for Efficient Polymer Solar Cells Advanced Materials 27:1170-1174 doi:10.1002/adma.201404317

Liu ZT, Wu Y, Zhang Q, Gao X (2016) Non-fullerene small molecule acceptors based on perylene diimides Journal of Materials Chemistry A 4:17604-17622 doi:10.1039/c6ta06978a

McAfee SM, Dayneko SV, Josse P, Blanchard P, Cabanetos C, Welch GC (2017) Simply Complex: The Efficient Synthesis of an Intricate Molecular Acceptor for High-Performance Air-Processed and Air-Tested Fullerene-Free Organic Solar Cells Chemistry of Materials 29:1309-1314 doi:10.1021/acs.chemmater.6b04862

McAfee SM, Topple JM, Hill IG, Welch GC (2015) Key components to the recent performance increases of solution processed non-fullerene small molecule acceptors Journal of Materials Chemistry $A$ 3:16393-16408 doi:10.1039/c5ta04310g

Mulligan CJ, Wilson M, Bryant G, Vaughan B, Zhou X, Belcher WJ, Dastoor PC (2014) A projection of commercial-scale organic photovoltaic module costs Solar Energy Materials and Solar Cells 120:9-17

Namazian M, Lin CY, Coote ML (2010) Benchmark Calculations of Absolute Reduction Potential of Ferricinium/Ferrocene Couple in Nonaqueous Solutions Journal of Chemical Theory and Computation 6:2721-2725 doi:10.1021/ct1003252

Nielsen CB, Holliday S, Chen H-Y, Cryer SJ, McCulloch I (2015) Non-Fullerene Electron Acceptors for Use in Organic Solar Cells Accounts of Chemical Research 48:2803-2812 doi:10.1021/acs.accounts.5b00199

Ren G, Ahmed E, Jenekhe SA (2011) Non-Fullerene Acceptor-Based Bulk Heterojunction Polymer Solar Cells: Engineering the Nanomorphology via Processing Additives Adv Energy Mater 1:946-953 doi:10.1002/aenm.201100285

Singh TB et al. (2005) High-mobility n-channel organic field-effect transistors based on epitaxially grown C 60 films Organic Electronics 6:105-110

Sonar P, Lim JPF, Chan KL (2011) Organic non-fullerene acceptors for organic photovoltaics Energy Environ Sci 4:1558-1574

Thompson BC, Fréchet JM (2008) Polymer-fullerene composite solar cells Angewandte chemie international edition 47:58-77

Wadsworth A et al. (2017) Highly Efficient and Reproducible Nonfullerene Solar Cells from Hydrocarbon Solvents ACS Energy Letters 2:1494-1500 doi:10.1021/acsenergylett.7b00390 
Wienk MM, Kroon JM, Verhees WJ, Knol J, Hummelen JC, van Hal PA, Janssen RA (2003) Efficient methano [70] fullerene/MDMO - PPV bulk heterojunction photovoltaic cells Angewandte Chemie 115:3493-3497

Zhao W, Li S, Yao H, Zhang S, Zhang Y, Yang B, Hou J (2017) Molecular Optimization Enables over 13\% Efficiency in Organic Solar Cells J Am Chem Soc 139: 7148-7151 